\title{
Characterization of the HD 17156 planetary system ${ }^{\star} \star \star \star$
}

\author{
M. Barbieri ${ }^{1}$, R. Alonso ${ }^{1}$, S. Desidera ${ }^{2}$, A. Sozzetti ${ }^{3}$, A. F. Martinez Fiorenzano ${ }^{4}$, J. M. Almenara ${ }^{5}$, M. Cecconi ${ }^{4}$, \\ R. U. Claudi ${ }^{2}$, D. Charbonneau ${ }^{7}$, M. Endl ${ }^{8}$, V. Granata ${ }^{2,9}$, R. Gratton ${ }^{2}$, G. Laughlin ${ }^{10}$, \\ B. Loeillet ${ }^{1,6}$, and Exoplanet Amateur Consortium ${ }^{\star \star \star}$
}

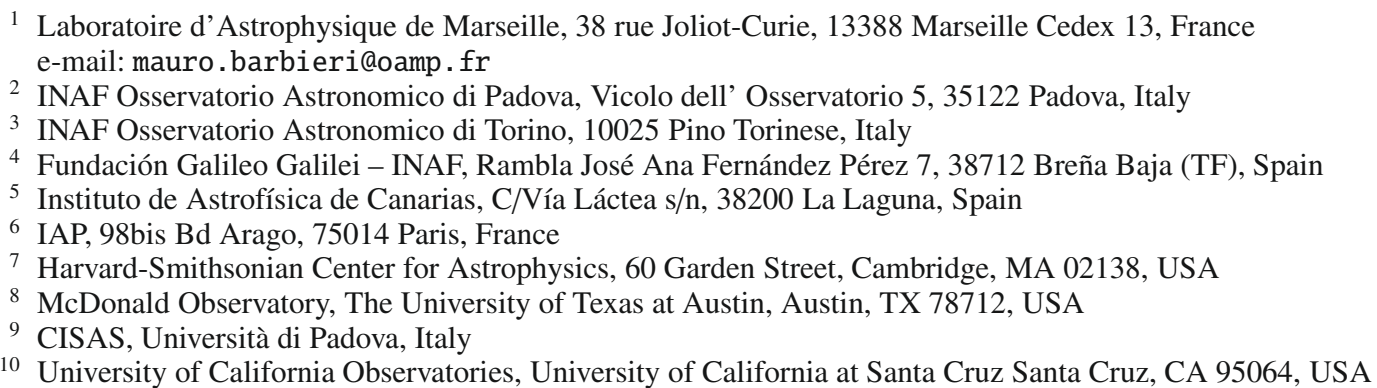

Received 2 December 2008 / Accepted 12 March 2009

\section{ABSTRACT}

\begin{abstract}
Aims. We present data to improve the known parameters of the HD 17156 system (peculiar due to the eccentricity and long orbital period of its transiting planet) and constrain the presence of stellar companions.

Methods. Photometric data were acquired for 4 transits, and high precision radial velocity measurements were simultaneously acquired with the SARG spectrograph at TNG for one transit. The template spectra of HD 17156 was used to derive effective temperature, gravity, and metallicity. A fit of the photometric and spectroscopic data was performed to measure the stellar and planetary radii, and the spin-orbit alignment. Planet orbital elements and ephemeris were derived from the fit. Near infrared adaptive optic images were acquired with the AdOpt module of TNG.

Results. We found that the star has a radius of $R_{\mathrm{S}}=1.44 \pm 0.03 R_{\odot}$ and the planet $R_{\mathrm{P}}=1.02 \pm 0.08 R_{\mathrm{J}}$. The transit ephemeris is $T_{\mathrm{c}}=2454756.73134 \pm 0.00020+N \cdot 21.21663 \pm 0.00045 \mathrm{BJD}$. Analysis of the Rossiter-Mclaughlin effect shows that the system is spin orbit aligned with an angle $\beta=4.8^{\circ} \pm 5.3^{\circ}$. The analysis of high resolution images did not reveal any stellar companion with a projected separation between of 150 and 1000 AU from HD 17156.
\end{abstract}

Key words. stars: individual: HD 17156 - stars: binaries: eclipsing - stars: planetary systems - techniques: spectroscopic techniques: photometric

\section{Introduction}

The discovery of transiting extrasolar planets (TESP) is of special relevance for the study and characterization of planetary systems. The combination of photometric and radial velocity measurements allows one to directly measure the mass and radius of an exoplanet, and hence its density, which is the primary constraint on a planet's bulk composition. Dedicated follow-up observations of TESP during primary transit and secondary eclipse

\footnotetext{
* Based on observations made with the Italian Telescopio Nazionale Galileo (TNG) operated on the island of La Palma by the Fundacion Galileo Galilei of the INAF (Istituto Nazionale di Astrofisica) at the Spanish Observatorio del Roque de los Muchachos of the Instituto de Astrofisica de Canarias. Based on observations collected at Asiago observatory, at Observatoire de Haute Provence and with Telast at IAC.

$\star \star$ Photometry and radial velocity data are only available in electronic form at the CDS via anonymous ftp to cdsarc.u-strasbg. fr $(130.79 .128 .5)$ or via

http://cdsweb.u-strasbg.fr/cgi-bin/qcat?J/A+A/503/601

$\star \star \star$ E.A.C. observations obtained by: F. Castellani (Mt. Baldo Observatory), B. Gary, J. Gregorio, C. Lopresti, A. Marchini (Siena University Observatory), M. Nicolini (Cavezzo Observatory), R. Papini, C. Vallerani.
}

at visible as well as infrared wavelengths allow direct measurements of planetary emission and absorption (e.g., Charbonneau et al. 2007, and references therein). Transmission spectroscopy during primary eclipse in recent years has been successful in characterizing the atmospheric chemistry of several Hot Jupiters (e.g., Charbonneau et al. 2002; Tinetti et al. 2007). Infrared measurements gathered at a variety of orbital phases, including secondary eclipse, have permitted the characterization of the longitudinal temperature profiles of nearby TESP. The rapidly increasing amount of high-quality data obtained for TESP has provided the first crucial constraints on theoretical models describing the physical structure and the atmospheres of gas and ice exoplanets. The detailed characterization of TESP ultimately is of special relevance to test several proposed formation and orbital evolution mechanisms of close-in planets.

The planet HD 17156b, detected by (Fischer et al. 2007, hereinafter F07) using the radial velocity method, was shown to transit in front of its parent star by Barbieri et al. (2007). Additional photometric measurements were presented in followup papers by Gillon et al. (2008), Narita et al. (2008), Irwin et al. (2008), Winn et al. (2008). This planet is unique among the known transiting systems in that its period (21.2 days) is more 
than 5 times longer than the average period for this sample, and it has the largest eccentricity $(e=0.67)$.

Schlesinger (1910), Rossiter (1924) and McLaughlin (1924) showed that a transiting object, such asr companion, produces a distortion in the stellar line profiles due to the partial eclipse of the rotating stellar surface during the event, and thus an apparent anomaly in the measured radial velocity of the observed primary star. In the case of close-in giant planets transiting solar-type stars, the amplitude of the Rossiter-McLaughlin effect ranges typically between a few and $\sim 100 \mathrm{~m} \mathrm{~s}^{-1}$, depending on orbital period, stellar and planetary radii, and stellar rotational velocity. This is within the current instrument capabilities for the brightest transiting planets. Indeed, this effect has been previously observed on several TESP (e.g. Winn 2008, and references therein). The observation of the Rossiter-McLaughlin effect allows us to measure the relative inclination angle between the sky projections of the orbital plane and the stellar spin axis. Another important result derived from these observations is the possibility to determine whether short orbital period transiting planets move in the same direction of the stellar spin, indicating the existence of strong dynamical interactions between the parent star and its planet. Measurements of the Rossiter-McLaughlin effect thus provide relevant residual evidence of planet formation and migration processes, as well as dynamical interactions with perturbing bodies (Marzari \& Weidenschilling 2002).

Up to now 8 of the 9 transiting planets for which the RossiterMcLaughlin effect was measured are coplanar systems, as our Solar System (the orbital axes of all the planets and the Sun spin axis are aligned within a few degrees). This is compatible with a formation mechanism for close-in giant planets including migration via tidal interactions with the protoplanetary disk. Only the XO-3 planet seems to be a non aligned system (Hébrard et al. 2008).

The measurement of the Rossiter-McLaughlin effect is of special relevance for the HD 17156 system, given the very high eccentricity and orbital period, much longer than the other transiting planets. With a period of 21 days, HD $17156 \mathrm{~b}$ is well outside the peak in the period distribution of close-in planets at about 3 days, possibly implying a different migration history with respect to the other transiting planets. An eccentricity as high as that of HD 17156b hardly can be explained by models of planet migration via tidal interactions with the protoplanetary disk. Alternatively, high-eccentricity planets might be the outcome of a variety of possible planet-planet dynamical interactions (Marzari \& Weidenschilling 2002). In these scenarios, high relative inclinations between the stellar rotation axis and the planet orbital plane are possible. An additional way to obtain large relative inclinations and eccentricities is represented by Kozai resonances with a close stellar companion. Interestingly, a rather large planetary mass coupled with a short orbital period might be an indication of binarity, as typically high-mass shortperiod planets occur in binary systems (Desidera \& Barbieri 2007).

Narita et al. (2008) presented the first observations of the Rossiter-McLaughlin effect for HD 17156. They found an angle between the sky projections of the orbital axis and the stellar rotation axis $\beta=-62 \pm 25^{\circ}$. However, Cochran et al. (2008) did not confirm this claim, suggesting instead well-aligned axes. Such a discrepancy calls for additional high-precision RV monitoring during planetary transit. These are presented in this work, along with additional photometric observations and a critical revision of stellar and planetary parameters. To further constrain the origin of the special properties of HD 17156b, we also
Table 1. Differential radial velocities and bisector velocity span of HD 17156 obtained with SARG at TNG.

\begin{tabular}{rrrrr}
\hline \hline BJD & $\begin{array}{r}\text { RV } \\
\mathrm{m} / \mathrm{s}\end{array}$ & $\begin{array}{r}\text { Err } \\
\mathrm{m} / \mathrm{s}\end{array}$ & $\begin{array}{r}\text { Span } \\
\mathrm{m} / \mathrm{s}\end{array}$ & $\begin{array}{r}\text { Err } \\
\mathrm{m} / \mathrm{s}\end{array}$ \\
\hline 2454398.58856 & -81.03 & 5.76 & & \\
2454438.40203 & 100.47 & 4.55 & -25.47 & 63.07 \\
2454438.41331 & 82.40 & 4.56 & -32.78 & 62.90 \\
2454438.42460 & 76.72 & 4.55 & 19.04 & 60.32 \\
2454438.43608 & 90.10 & 4.43 & 74.70 & 59.30 \\
2454438.44737 & 91.46 & 3.98 & -5.58 & 56.75 \\
2454438.45866 & 76.28 & 4.35 & 64.69 & 59.89 \\
2454438.47011 & 65.86 & 3.98 & 47.68 & 57.10 \\
2454438.48140 & 60.08 & 4.01 & 40.49 & 53.96 \\
2454438.49270 & 30.74 & 3.72 & 119.82 & 57.44 \\
2454438.50415 & 32.97 & 3.98 & 216.98 & 59.74 \\
2454438.51544 & 20.63 & 4.49 & 108.08 & 57.92 \\
2454438.52671 & 18.78 & 4.59 & 74.54 & 57.66 \\
2454438.53816 & 4.29 & 4.29 & 82.66 & 58.07 \\
2454438.54946 & -3.77 & 4.13 & 90.90 & 57.79 \\
2454438.56075 & 7.21 & 4.93 & 41.59 & 63.36 \\
2454438.57218 & -3.84 & 3.77 & 58.41 & 57.50 \\
2454438.58349 & -11.52 & 4.53 & 50.75 & 60.76 \\
2454438.59478 & -31.71 & 4.43 & 45.47 & 59.96 \\
2454438.62486 & -26.61 & 4.74 & 36.70 & 64.19 \\
2454438.63615 & -43.01 & 4.01 & 18.82 & 58.87 \\
2454438.64745 & -46.79 & 4.23 & 66.00 & 65.44 \\
2454438.65894 & -54.19 & 4.77 & -38.79 & 65.29 \\
2454438.67023 & -55.35 & 4.72 & 3.19 & 67.30 \\
2454438.68152 & -64.85 & 5.32 & 102.93 & 70.23 \\
2454438.69306 & -71.94 & 5.20 & 73.98 & 67.86 \\
2454438.70435 & -82.48 & 5.08 & 111.26 & 72.47 \\
2454438.71584 & -88.29 & 5.13 & -18.92 & 72.31 \\
2454438.72713 & -92.59 & 5.15 & 94.27 & 72.55 \\
\hline
\end{tabular}

searched for possible wide stellar companions using adaptive optics observations.

The overall organization of this paper begins in Sect. 2 with the description of high resolution spectroscopy data obtained with the SARG spectrograph at TNG. Section 3 covers the analysis of the stellar parameters. Section 4 presents the photometric data collected during several planetary transits. Section 5 describes the analysis of the radial velocity and photometric data, and their results. In Sect. 6 we present the results of the search for additional stellar companions to HD 17156. In Sect. 7 our conclusion are presented.

\section{High resolution spectroscopy}

We observed HD 17156 on 2007 December 3, including continuous monitoring (about 8 hours) during the transit, with SARG, the high resolution spectrograph of the TNG (Gratton et al. 2001). Observing conditions were not optimal, with seeing ranging from $1.3^{\prime \prime}$ to $2.0^{\prime \prime}$. We obtained the stellar template (without the iodine cell) first and then started uninterrupted series of observations with the iodine cell. The exposure time of the spectra acquired with the iodine cell was $900 \mathrm{~s}$, typically resulting in a $\mathrm{S} / \mathrm{N}$ of about 80 per pixel. One additional spectrum was obtained on 25 Oct. 2007. These spectra were reduced and analyzed in the same way those from the ongoing planet search program with SARG (Desidera et al. 2007) using the AUSTRAL code (Endl et al. 2000). Table 1 lists the radial velocities.

The absolute radial velocity of HD 17156 was derived by cross correlating the stellar template acquired with SARG to a few suitable reference stars observed with the same set-up 
and with available high-accuracy absolute radial velocity from Nidever et al. (2002). It results in $-3.15 \pm 0.20 \mathrm{~km} \mathrm{~s}^{-1}$.

\section{Stellar parameters}

\subsection{Spectroscopic analysis}

We have used the TNG/SARG template spectrum of HD 17156 to provide an independent assessment of its atmospheric parameters $\left(T_{\text {eff }}, \log g\right.$, and $\left.[\mathrm{Fe} / \mathrm{H}]\right)$ with respect to the values reported by $\mathrm{F} 07$.

Our methodology follows a standard procedure whose details can be found in several works (e.g., Gonzalez \& Lambert 1996; Gonzalez et al. 2001; Santos et al. 2004). We briefly summarize it here. We initially selected a set of relatively weak FeI and Fe II lines (see, e.g., Sozzetti et al. 2004, and references therein, for details on the line list), and measured equivalent widths (EWs) using the automated software ARES ${ }^{1}$, made available to the community by Sousa et al. (2007). The EWs measured with ARES are then entered in to the 2002 version of the MOOG spectral synthesis code (Sneden 1973$)^{2}$, together with a grid of Kurucz ATLAS plane-parallel stellar model atmospheres (Kurucz 1993).

The atmospheric parameters of HD 17156 are then derived under the assumption of local thermodynamic equilibrium, using the standard technique of Fe ionization balance (see, e.g., Santos et al. 2004; Sozzetti et al. 2004, and references therein). We obtained $T_{\text {eff }}=6100 \pm 75 \mathrm{~K}, \log g=4.1 \pm 0.1$, and $[\mathrm{Fe} / \mathrm{H}]=$ $+0.14 \pm 0.08$, the formal errors on $T_{\mathrm{eff}}$ and $\log g$ having been derived using the procedure described in Neuforge-Verheecke \& Magain (1997) and Gonzalez \& Vanture (1998), while the nominal uncertainty for $[\mathrm{Fe} / \mathrm{H}]$ corresponds to the scatter obtained from the FeI lines rather than the formal error of the mean.

We also quantified the sensitivity of our iron abundance determination to variations of $\pm 1 \sigma$ with respect to the nominal $T_{\text {eff }}$ and $\log g$ values, and found changes in $[\mathrm{Fe} / \mathrm{H}]$ of at most 0.05 dex, below the adopted uncertainty of 0.08 dex.

To further test the accuracy of the $T_{\text {eff }}$ determination above, we have carried out additional consistency checks. For example, in Fig. 1 we show the comparison of the observed $\mathrm{H}_{\alpha}$ line profile in an archival Keck/HIRES spectrum against four synthetic profiles for solar-metallicity dwarfs $([\mathrm{Fe} / \mathrm{H}]=0.0, \log g=4.5)$ from the Kurucz database. As is well-known, the $\mathrm{H}_{\alpha}$ line is very sensitive to changes in $T_{\text {eff }}$, while relatively insensitive to changes in $\log g$ and $[\mathrm{Fe} / \mathrm{H}]$ (see, e.g., Santos et al. 2006; Sozzetti et al. 2007, and references therein), thus this exercise helps to test the accuracy of the spectroscopic $T_{\text {eff }}$ derived above. The results shown in Fig. 1, in which a $10 \AA$ region centered on $\mathrm{H}_{\alpha}$ is displayed together with four calculated profiles for different $T_{\text {eff }}$ values, indicate rather good agreement with the estimate reported in Table 2.

\subsection{Age}

Based on isochrone fitting, F07 reported an age estimate for HD 17156 of $5.7_{-1.9}^{+1.3}$ Gyr, suggesting an old, slightly evolved F8/G0 primary. We performed an independent isochrone fitting using the set of isochrones of Girardi et al. (2000) and the software PARAM described in da Silva et al. $(2006)^{3}$. The input values for PARAM are the parallax, the visual magnitude, $[\mathrm{Fe} / \mathrm{H}]$

\footnotetext{
${ }^{1}$ http://www.astro.up.pt/ sousasag/ares

2 http://verdi.as.utexas.edu/moog.html

3 http://stev.oapd.inaf.it/param
}

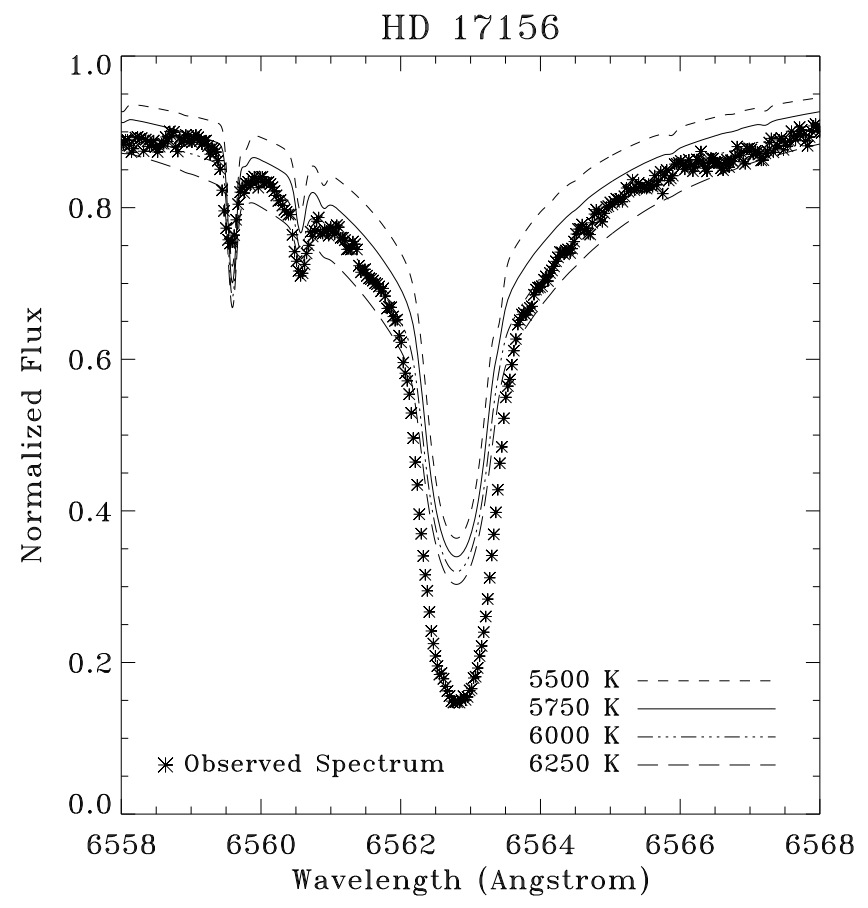

Fig. 1. The portion of the spectrum of HD 17156 around $\mathrm{H}_{\alpha}$ and synthetic profiles for four different $T_{\text {eff }}$. A $T_{\text {eff }}$ close to $6000 \mathrm{~K}$ is suggested by the fit of the wings of the line.

and $T_{\text {eff. We used the parallax from van Leeuwen (2007) and }}$ for the metallicity and effective temperature we ran the code twice, once with the values from F07 and once with our estimate. The results of PARAM for the stellar age are $2.4 \pm 1$ and $2.8 \pm 1$ Gyr, for the F07 and our parameters, respectively. These are only marginally compatible with the previous age estimate by F07. The stellar mass and radius are instead fully compatible (see below).

Other indirect age indicators confirm an age of a few Gyr. The low level of Ca II H\&K chromospheric activity suggests an age of about 6 Gyr (Fischer et al. 2007). The lack of X-ray emission from ROSAT (Voges et al. 2000) yields an upper limit of $\log L_{X}<28.7$. This in turn implies an age older than $1.6 \mathrm{Gyr}$, using the age-X ray emission calibration by Mamajek \& Hillenbrand (2008).

To obtain an additional age estimate and to investigate possible chemical peculiarities of HD 17156 with respect to other planet hosts with similar physical properties, we measured its lithium (Li) abundance.

Figure 2 shows a spectral synthesis of a $10 \AA$ region centered on the Li $\lambda=6707.8 \AA$ line in an archival Keck/HIRES spectrum of HD 17156, and using the atmospheric parameters derived from the Fe-line analysis and the line list of Reddy et al. (2002). In the figure, the observed spectrum is compared to three synthetic spectra, each differing only in the assumed Li abundance. We find a best-fit value of $\log \epsilon(\mathrm{Li}) \approx 2.80$ for HD 17156 . We then infer a rather old age for the star of $t>2 \mathrm{Gyr}$, based on the average $\mathrm{Li}$ abundance curves as a function of effective temperature for clusters of different ages reported by Sestito \& Randich (2005).

The measured Li abundance for HD 17156 does not appear peculiar when compared to that of sub-samples of nearby planet hosts with similar $T_{\text {eff }}$ (Israelian et al. 2004; Gonzalez 2008). To further investigate these issues we will present in a future paper a more detailed study of the elemental abundances in HD 17156. 
Table 2. Upper panel: input parameters for the Monte Carlo experiment and best values. Lower panel: kinematical properties and galactic orbit parameters.

\begin{tabular}{|c|c|c|}
\hline \multicolumn{3}{|c|}{$\begin{array}{l}\text { Monte Carlo experiment } \\
\text { Input parameters }\end{array}$} \\
\hline parallax & $13.33 \pm 0.72$ & mas \\
\hline $\operatorname{mag} V$ & $8.172 \pm 0.031$ & mag \\
\hline $\operatorname{mag} K$ & $6.807 \pm 0.024$ & mag \\
\hline$T_{\text {eff }}$ & $6100 \pm 75$ & $\mathrm{~K}$ \\
\hline $\mathrm{BC}$ & $-0.03 \pm 0.02$ & mag \\
\hline \multicolumn{3}{|c|}{ Output parameters } \\
\hline$M_{\mathrm{bol}, \odot}$ & $3.69 \pm 0.12$ & mag \\
\hline$M_{V}$ & $3.73 \pm 0.12$ & mag \\
\hline$L$ & $2.68 \pm 0.28$ & $L_{\odot}$ \\
\hline$R$ (Stefan-Boltzmann) & $1.49 \pm 0.09$ & $R_{\odot}$ \\
\hline$R$ (Kervella) & $1.45 \pm 0.07$ & $R_{\odot}$ \\
\hline$M\left(\right.$ Malkov, $\left.M_{\mathrm{V}}\right)$ & $1.21 \pm 0.04$ & $M_{\odot}$ \\
\hline$M($ Malkov, $L)$ & $1.25 \pm 0.04$ & $M_{\odot}$ \\
\hline$M\left(M \propto L^{4.5}\right)$ & $1.22 \pm 0.04$ & $M_{\odot}$ \\
\hline$M\left(\right.$ Henry, $\left.M_{\mathrm{V}}\right)$ & $1.29 \pm 0.03$ & $M_{\odot}$ \\
\hline $\log g($ mean $)$ & $4.21 \pm 0.05$ & cgs \\
\hline$\rho($ mean $)$ & $0.57 \pm 0.10$ & $\mathrm{~g} / \mathrm{cm}^{3}$ \\
\hline \multicolumn{3}{|c|}{ Kinematical properties } \\
\hline RV & $-3.15 \pm 0.2$ & $\mathrm{~km} \mathrm{~s}^{-1}$ \\
\hline$\mu_{\alpha}$ & $91.14 \pm 0.49$ & mas/yr \\
\hline$\mu_{\delta}$ & $-33.14 \pm 0.56$ & mas/yr \\
\hline$U$ & $0.6 \pm 0.2$ & $\mathrm{~km} \mathrm{~s}^{-1}$ \\
\hline$V$ & $26.1 \pm 2.0$ & $\mathrm{~km} \mathrm{~s}^{-1}$ \\
\hline$W$ & $-22.8 \pm 1.5$ & $\mathrm{~km} \mathrm{~s}^{-1}$ \\
\hline$R_{\min }$ & $8.0 \pm 0.3$ & $\mathrm{kpc}$ \\
\hline$R_{\max }$ & $10.9 \pm 0.3$ & $\mathrm{kpc}$ \\
\hline$R_{\text {med }}$ & $9.5 \pm 0.4$ & $\mathrm{kpc}$ \\
\hline$Z_{\max }$ & $0.2 \pm 0.1$ & $\mathrm{kpc}$ \\
\hline$e$ (galactic orbit) & $0.15 \pm 0.05$ & \\
\hline
\end{tabular}

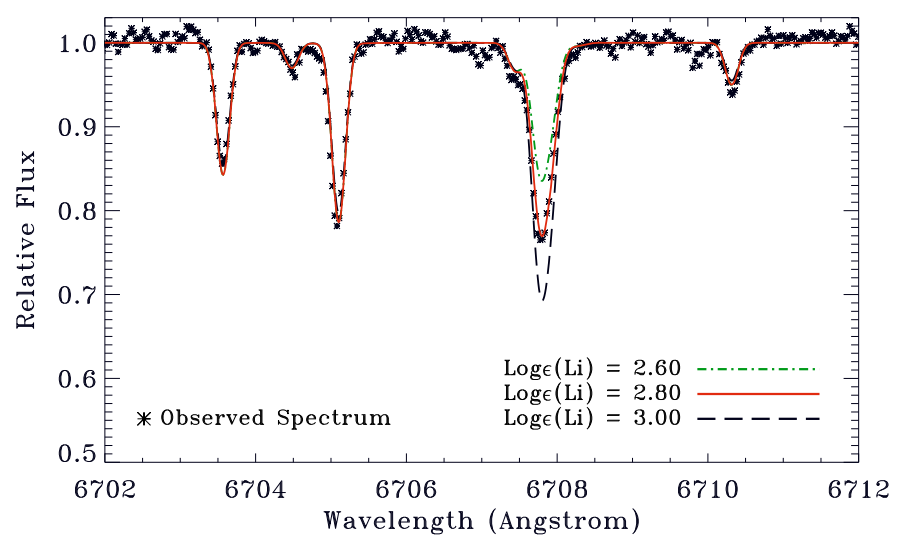

Fig. 2. Portion of the HD 17156 spectrum centered on the Lithium $6707.8 \AA$ line. The results of the spectral synthesis for three different lithium abundance are overplotted.

\subsection{Stellar mass and radius}

F07 provided mass and radius estimate from isochrone interpolations (mass $1.2 \pm 0.1 M_{\odot}$, radius $1.47_{-0.17}^{+0.13} R_{\odot}$ ). Our isochrone fit (see Sect. 3.2) gives $1.21 \pm 0.03$ and $1.19 \pm 0.03 M_{\odot}$ when assuming $T_{\text {eff }}$ and $[\mathrm{Fe} / \mathrm{H}]$ from $\mathrm{F} 07$ and our analysis respectively, and same the radius $1.35 \pm 0.08 R_{\odot}$.

The comparison of the results of the two fits shows that they are compatible within error bars, but the best values are slightly different. The origin of these differences is not clear, and to understand this problem independent measurements of the star mass and radii are needed.

\subsubsection{Monte Carlo experiment. Method}

For this purpose we adopted an approach based on a Monte Carlo experiment. In each realization we generated a set of observed stellar properties. Using calibrations from the literature we obtained the corresponding values for mass and radius. From the resulting distributions we obtained the most probable values and relative errors for the mass and radius.

In more detail, we created $10^{6}$ different synthetic systems where we generated Gaussian distributions for the parallax, the $V$ and $K$ magnitudes, and $T_{\text {eff }}$, using as standard deviations their respective error bars. The input parameters and their relative standard deviations are reported in Table 2. In the same way, we generated values for the bolometric correction and the bolometric magnitude of the Sun. For each synthetic set obtained this way we calculated the absolute magnitudes, luminosity, radius, mass, density and gravity.

The $V$ magnitude was obtained from Simbad, $K$ magnitude from 2MASS after conversion to the Bessel-Brett system (Carpenter 2001), and the $T_{\text {eff }}$ from our spectroscopic determination. For the parallax we adopted the recently revised Hipparcos value (van Leeuwen 2007), which indicates that HD 17156 lies at $75 \mathrm{pc}$ from the Sun, 3 pc closer than the previous estimate. The bolometric correction was set to $\mathrm{BC}=-0.03$ (Girardi et al. 2000) while for the solar bolometric magnitude we used the value $M_{\text {bol, } \odot}=4.77 \pm 0.02$.

The stellar radius was obtained using the $T_{\text {eff }}-\mathrm{K}$ mag calibration of Kervella et al. (2004), and using the StefanBoltzmann law. The stellar mass was calculated from various mass-luminosity relations (MLR) namely: $i$ ) the classical MLR $L \propto M^{4.5}$; ii) the MLR of Malkov (2007) using absolute magnitude and iii) stellar luminosity; iv) the MLR of Henry (2004). Density and gravity were estimated directly from the radius and mass assuming a spherically symmetric star.

\subsubsection{Monte Carlo experiment. Results}

The distribution of absolute magnitudes in $V$ band peaks at 3.7 (Fig. 3, upper left), while the luminosity peak at $2.5 L_{\odot}$ (Fig. 3, upper right). These values are slightly different from the ones obtained by Fischer et al. (2007), and the source of these differences is ascribed only to the difference in the adopted parallax.

The resulting distributions for the radius are shown in Fig. 3 (middle left). The two relations used provide similar results $\left(R_{\mathrm{S}} \sim 1.4 R_{\odot}\right)$ for the best value and also the shape of the distributions is very similar. Fischer et al. (2007) suggest a slightly larger radius. Also in this case, the difference originates from the change in the adopted parallax.

In Fig. 3 (middle right) we present the mass distributions obtained with the MLRs. Using the relation of Henry (2004) we obtain the highest mass $\left(M \sim 1.28 M_{\odot}\right)$. However, we note that this relation was originally derived from parameters of close binary stars. Malkov (2003) demonstrated that this kind of relation does not describe single stars well. In order to avoid this problem we adopted the MLRs of Malkov (2007) obtained on detached main-sequence double-lined eclipsing binaries. These relations are also valid for slightly evolved stars like HD 17156 (almost all the stars used to derive these relations are also slightly evolved; O. Malkov, private communication). We obtain best values for the mass between 1.2 and $1.24 M_{\odot}$, the first obtained using $\operatorname{MLR}\left(M_{V}\right)$ and the second using $\operatorname{MLR}(L)$. The classical MLR $\left(M \propto L^{4.5}\right)$ provides $M \sim 1.22 M_{\odot}$. These results are consistent with the values estimated by F07 $\left(1.2 \pm 0.1 M_{\odot}\right)$. 

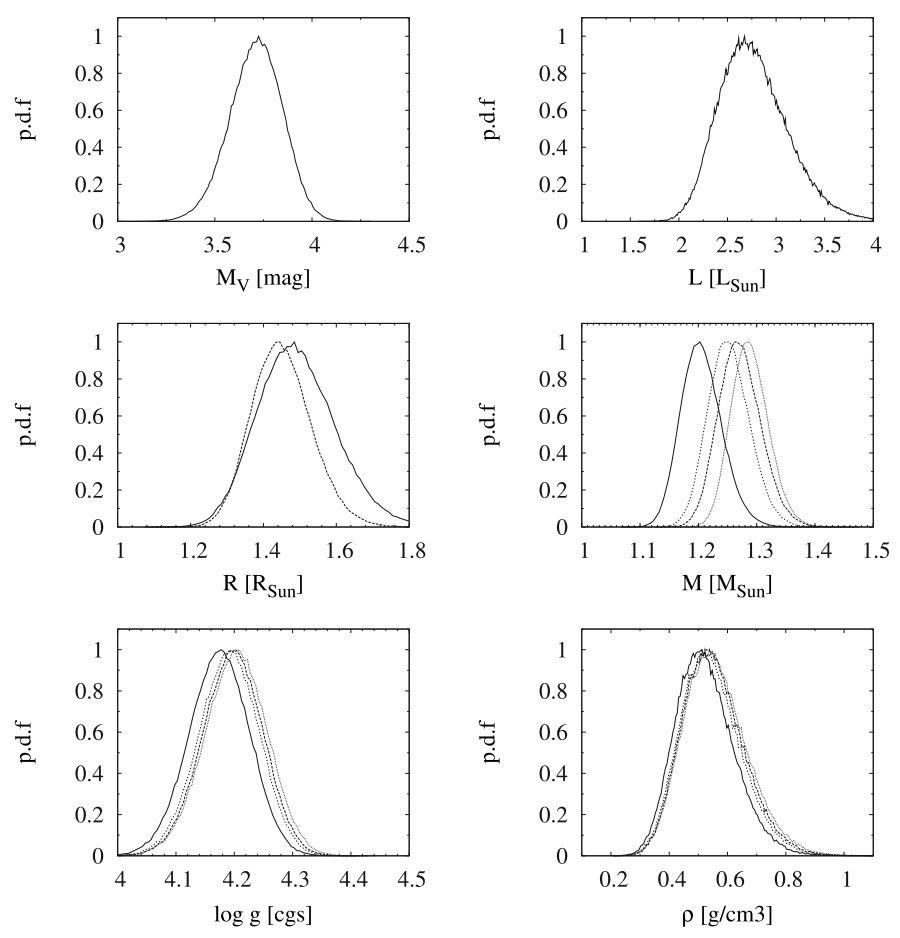

Fig. 3. Probability density function of the stellar parameters obtained with the Monte Carlo experiment. Upper left: absolute magnitude. Upper right: luminosity. Middle left: radius: thick line results using Stefan-Boltzmann law, thin line results using the calibration of Kervella $\left(T_{\text {eff }}, M_{K}\right)$. Middle right: mass, from left to right results using: Malkov $\operatorname{MLR}\left(M_{V}\right), M \propto L^{4.5}$, Malkov MLR $(L)$, and Henry $\operatorname{MLR}\left(M_{V}\right)$. Lower left: $\log g$. Lower right: density.

The resulting distributions for the gravity and the density are portrayed in the lower panels of Fig. 3. The mean values are $\log g=4.22$ and $\rho=0.58 \mathrm{~g} / \mathrm{cm}^{3}$.

The results of this experiment show a fairly good agreement with the estimate of $R_{\mathrm{S}}$ based on isochrone fitting (both ours and the one by F07). We conclude that the two independent approaches based on isochrone fitting and the use of scaling relations provide consistent results.

In the following we do not adopt a value for the radius, because we want to determine its value independently from the light-curve fits. Moreover, we fix the value of the mass to the value of the weighted mean of our mass estimation (not using the Henry MLR results) $M=1.24 \pm 0.03 M_{\odot}$. We summarize in Table 2 all the data relative to this Monte Carlo experiment.

\subsection{Rotational velocity}

F07 derived vsin $i_{\mathrm{S}}=2.6 \pm 0.5 \mathrm{~km} \mathrm{~s}^{-1}$ for HD 17156. Our template spectrum is suitable for an independent measurement of this quantity. We discuss here three different methods adopted for the measurement of $v \sin i_{\mathrm{S}}$, based on our template spectra.

With the first method we derive $v \sin i_{\mathrm{S}}$ by the Fast Fourier Transform analysis of the star's absorption profile (see Fig. 4). To determine the $v \sin i_{\mathrm{S}}$, the observed profile of a stellar absorption line is made symmetric by mirroring one of its halves, with the purpose of reducing the noise of the FFT. A new profile is calculated by the convolution of a macroturbulence profile (Gaussian) and a rotational one, to compare the FFTs of the symmetric and the calculated (model) profiles. The $v \sin i_{\mathrm{S}}$ value of the rotation profile is set as variable parameter until the first minimum of the FFT from the calculated profile coincides with

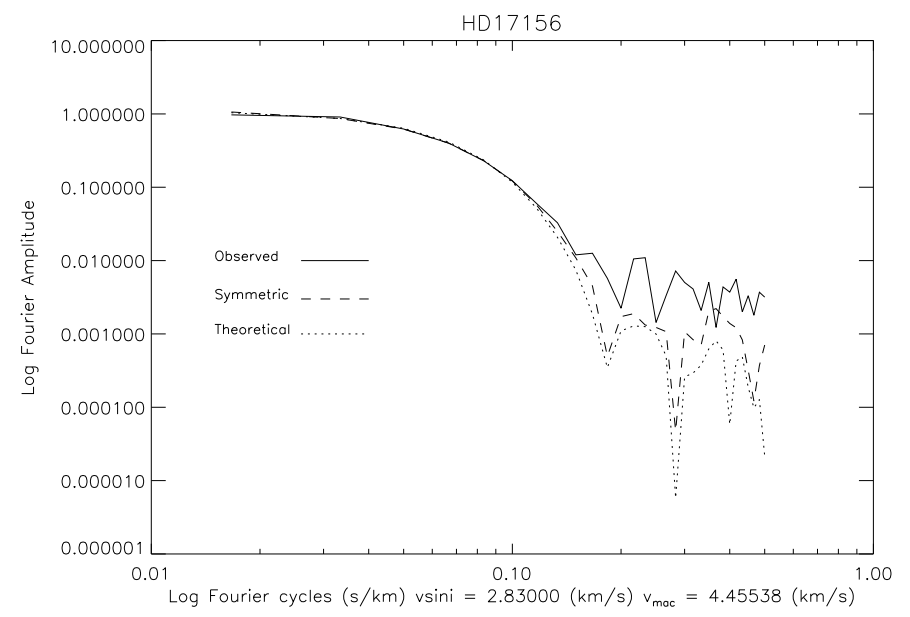

Fig. 4. Estimation of $v \sin i_{\mathrm{S}}$ through the FFT. Three profiles were analyzed: the observed profile (solid line), the symmetric profile (dashed line) mirroring one half of the profile, and a theoretical profile (dotted line). The FFT corresponds to a case considering $T_{\text {eff }}=6079 \mathrm{~K}$ and Eq. (1) given by (Valenti \& Fischer 2005).

the minimum of the FFT from the symmetric one. The value of $v \sin i_{\mathrm{S}}$ for HD 17156 was determined considering possible values of macroturbulent velocity $\left(v_{\text {mac }}\right)$ from B-V and $T_{\text {eff }}$ (Valenti $\&$ Fischer 2005), and we obtained a $v \sin i_{\mathrm{S}}$ ranging from 1.8 to $2.8 \mathrm{~km} \mathrm{~s}^{-1}$.

The second method that we used consists of obtaining the rotational velocity by means of a suitable calibration of the FWHM of the cross-correlation function against the $B-V$ color. This relation was derived for all the stars in the SARG planet search survey, and it was calibrated into $v \sin i$ using stars with known rotational velocity from the literature. Using the $B-V$ from the Tycho catalog converted to the Johnson system $(B-V=0.632)$, the resulting $v \sin i_{\mathrm{S}}=3.2 \pm 1 \mathrm{~km} \mathrm{~s}^{-1}$.

Finally, using MOOG we synthesized a number of isolated FeI lines in the template spectrum. From these we measured $v \sin i_{\mathrm{S}}=3.0 \pm 0.5 \mathrm{~km} \mathrm{~s}^{-1}$.

The values obtained with the three methods suggest a range of values for $v \sin i_{\mathrm{S}}$ compatible with the measurement of F07. The measurement of $v \sin i_{\mathrm{S}}$ from the analysis of the RossiterMclaughlin effect will be presented in Sect. 5.3.

\subsection{Galactic orbit}

The measurements of the absolute radial velocity given in Sect. 2, together with the revised parallax and proper motion from Hipparcos (van Leeuwen 2007), allow one to calculate the space velocity with respect to the Local Standard of Rest and the galactic orbit of HD 17156. Space velocities are calculated following the procedure delineated by Johnson \& Soderblom (1987) and Murray (1989), adopting the value of standard solar motion of Dehnen \& Binney (1998) (with $U$ positive toward the galactic anticenter). The calculations yield $(U, V, W)=$ $(+0.6,+26.1,-22.8) \mathrm{km} \mathrm{s}^{-1}$. The galactic orbit of the star is obtained integrating the equations of motion of a massless particle in the potential described by Allen \& Santillan (1991). The equations of motion are solved using the RADAU integrator (Everhart 1985) assuming that the rectangular galactocentric coordinates of the Sun are $\left(X_{\odot}, Y_{\odot}, Z_{\odot}\right)=(8.0,0.0,0.015) \mathrm{kpc}$, and that the local circular velocity is $220 \mathrm{~km} \mathrm{~s}^{-1}$. We compute 1000 orbits each time randomly varying the initial coordinates and velocity of the star within the error bars and integrating for 
Table 3. Summary of photometric observations of HD 17156 performed in 2007/08.

\begin{tabular}{l|l|l|l|l}
\hline \hline Site/Observer & $\begin{array}{l}\text { Diameter } \\
\mathrm{m}\end{array}$ & $\begin{array}{l}\text { rms OOT } \\
\mathrm{mmag}\end{array}$ & Date & $\begin{array}{l}T_{\mathrm{c}} \\
\text { BJD }\end{array}$ \\
\hline Telast & 0.30 & 4.2 & 2007 September 10 & $2454353.6130 \pm 0.0200$ \\
Gasparri & 0.20 & 5.3 & & \\
Lopresti & 0.18 & 7.0 & & \\
\hline Asiago & 1.82 & 2.8 & 2007 December 03 & $2454438.4745 \pm 0.0005$ \\
OHP & 1.2 & 2.0 & & \\
Telast & 0.3 & 11.2 & & \\
Obs. Cavezzo & 0.4 & 6.1 & & \\
Lopresti & 0.18 & 10.5 & & \\
Obs. Univ.Siena & 0.3 & 8.4 & & \\
Obs. Mt. Baldo & 0.4 & 7.3 & & \\
Papini & 0.3 & 6.9 & & \\
Vallerani & 0.25 & 5.9 & & \\
\hline Gary & 0.3 & 5.6 & 2007 December 25 & $2454459.6909 \pm 0.0032$ \\
\hline Gregorio & 0.3 & 3.8 & 2008 September 25 & $2454735.5135 \pm 0.0027$ \\
\hline Gary & 0.3 & 8.9 & 2008 October 17 & $2454756.7313 \pm 0.0024$ \\
\hline
\end{tabular}

The last column report the midtransit times for each date obtained from the fit in Sect. 5.2.

a time of $4 \mathrm{Gyr}(\approx 15$ full orbits around the Galactic Center). The $U V W$ spatial velocity and the mean values of the computed orbits are reported in Table 2. The mean galactic radius provides an estimate of the galactocentric distance of the star at the moment of its formation. The value for HD $17156, R_{\text {med }}=9.5 \mathrm{kpc}$, implies that the star spends most of its time in regions outside of the solar circle.

\section{Photometric observations}

Photometric observations of the transit of 2007 December 3 were obtained with various telescopes. On 2007 December 2 we used three medium-class telescopes (Asiago $1.82 \mathrm{~m}$ and OHP $1.20 \mathrm{~m}$ ) and a number of small telescopes, including six $30-40 \mathrm{~cm}$ amateur-operated telescopes all located in continental Europe as well as the Telast 0.3 -m telescope in the Canary Islands. Weather conditions across continental Europe were not optimal.

Observations at Asiago started under photometric conditions, transit ingress was observed but observations ended on December 04 UT 02:30, due to the presence of clouds, which prevented the observations of the third and fourth contacts. OHP observations were performed under variable sky conditions due to intermittent clouds and veils; good observing conditions were achieved only during the transit time window, with only a small coverage of the Out Of Transit (OOT) flat part of the lightcurve before the first contact. Observations with Telast were obtained under normal sky conditions and were performed throughout the night. Amateur observations were carried out by six observatories spread over central and northern Italy. Observing conditions suffered from clouds and veils similarly to the other European sites involved in the campaign, and the full transit was successfully observed by four telescopes, the remaining two observatories obtaining data only for the ingress phase of the transit.

Three additional attempts to coordinate transit observations of HD 17156b were carried out on 25 December 2007 and on September 25 and October 17 2008. Unfortunately, due to bad weather conditions, there was only one useful observation for each date.

All observations were obtained in $R$ band except the two obtained by Gary that was acquired in white light; the characteristics of the different telescopes are summarized in Table 3.

\subsection{Data reduction}

The Asiago, OHP, and Telast raw images were calibrated using flat field and bias frames. The resulting images were analyzed with IDL routines to perform standard aperture photometry. The center of the aperture was calculated using a Gaussian fit, and the aperture radius was held fixed for each set (in the range 15-20 pixels). The sky background contribution was removed after an estimation. The brightest non-variable stars in the field were measured in the same way, and a reference light curve was constructed by adding the flux of these stars. The target flux was divided by this reference to get the final normalized light-curve.

The images obtained by amateur telescopes were reduced, bias subtracted, and dark flat-field corrected using commercial software. Aperture photometry of HD 17156 was then performed using IRIS $^{4}$ and adopting a fixed aperture equal to 2 times the stellar FWHM. The sum of the flux of the brightest stars in the field was used as reference to build the normalized light-curve.

The final light-curve for each telescope was corrected for differential airmass and residual systematic effects by dividing them by a linear function of time to the region outside the transit. The photometric error on each point of the lightcurve was calculated as the rms over an interval of $30 \mathrm{~min}$ (the timescale of the ingress/egress phase). The typical rms values of the OOT lightcurves are reported in Table 3, while the complete photometric dataset is available in electronic format at CDS.

The whole dataset consists of $\sim 7000$ photometric points. We used these data to perform a global analysis of the planetary transit.

For the light curve fit we used all the lightcurves that we collected without performing data binning. In Fig. 5 we show the light curves used in this study, folded with the best orbital period from the fit. For displaying purposes the combined lightcurve of the 15 lightcurves is shown in Fig. 6. This combined light curve was obtained using a bin width of $90 \mathrm{~s}$; the OOT has an rms of 0.0016 .

\section{System parameters}

We performed the analysis of the HD 17156 system in three steps. First, using the radial velocities presented in Table 1 along

${ }^{4}$ http://www.astrosurf.com/buil/iris 

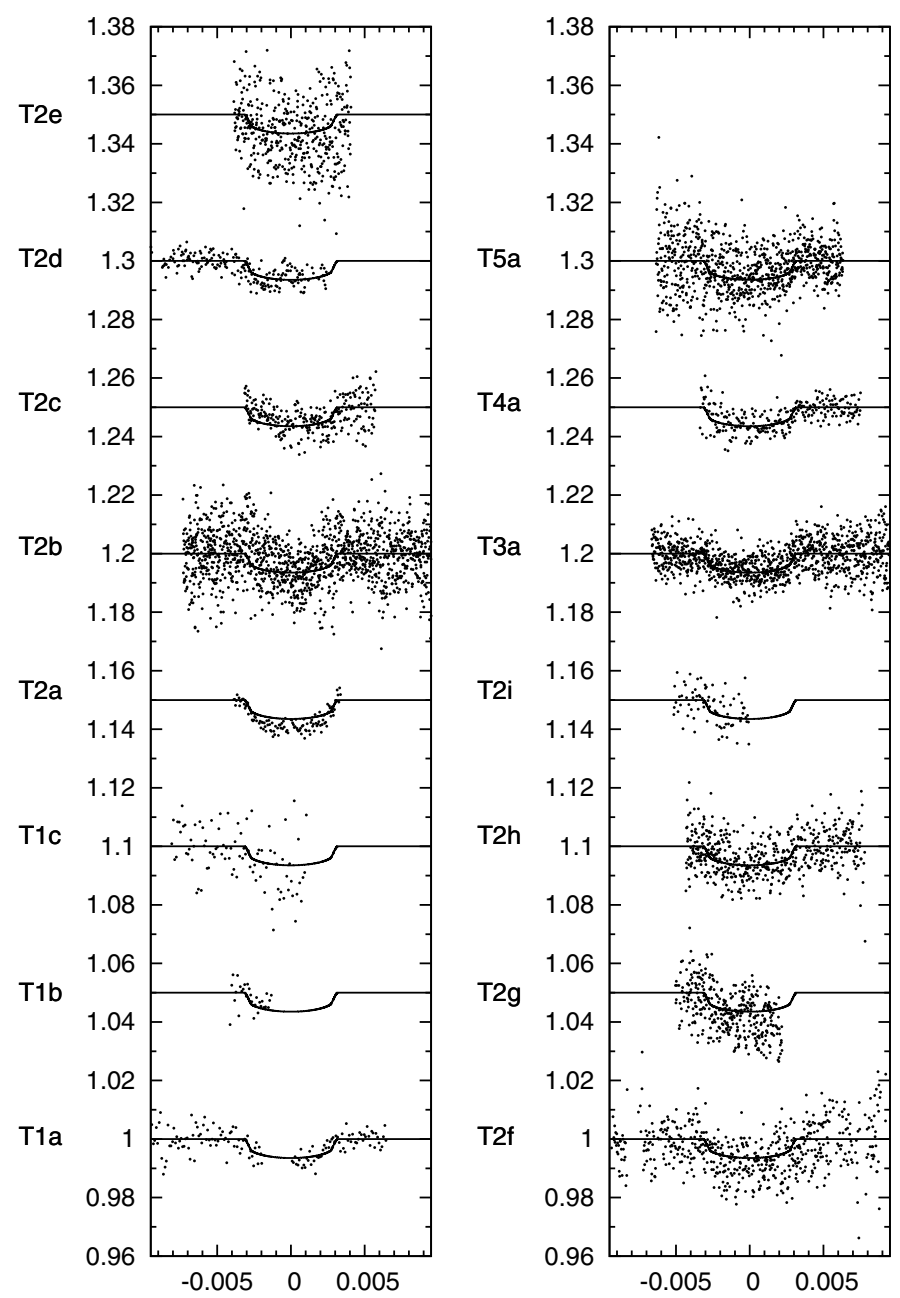

Table 4. Parameters of the HD 17156 system.

\begin{tabular}{lll}
\hline \hline & Orbital parameters & \\
$P$ & $21.21663 \pm 0.00045$ & day \\
$a$ & $0.1614 \pm 0.0022$ & $\mathrm{AU}$ \\
$e$ & $0.6819 \pm 0.0044$ & \\
$i$ & $87.89 \pm 0.11$ & $\mathrm{deg}$ \\
$\omega$ & $121.9 \pm 0.23$ & $\mathrm{deg}$ \\
$\beta$ & $4.8 \pm 5.6$ & $\mathrm{deg}$ \\
$K_{\mathrm{RV}}$ & $279.8 \pm 0.06$ & $\mathrm{~m} / \mathrm{s}$ \\
$T_{\mathrm{P}}$ & $2454757.00787 \pm 0.00298$ & $\mathrm{BJD}$ \\
$T_{\mathrm{c}}$ & $2454756.73134 \pm 0.00205$ & $\mathrm{BJD}$ \\
$k_{\mathrm{rr}}=R_{\mathrm{p}} / R_{\mathrm{S}}$ & $0.0727 \pm 0.0006$ & \\
$\theta_{1}$ & $-0.003145 \pm 0.000344$ & \\
$\mathrm{phase}$ egress & $0.003160 \pm 0.000344$ & \\
$\operatorname{transit}$ duration & $3.21 \pm 0.08$ & $\mathrm{hour}$ \\
\hline & \multicolumn{1}{c}{ Star parameters } & \\
$M_{\mathrm{S}}$ & $1.24 \pm 0.03$ & $M_{\odot}$ \\
$R_{\mathrm{S}}$ & $1.44 \pm 0.08$ & $R_{\odot}$ \\
$L$ & $2.58 \pm 0.36$ & $L_{\odot}$ \\
$\log g_{\mathrm{S}}$ & $4.22 \pm 0.05$ & $\mathrm{cgs}$ \\
$\rho_{\mathrm{S}}$ & $0.59 \pm 0.06$ & $\mathrm{~g} / \mathrm{cm}^{3}$ \\
$v \sin i_{\mathrm{S}}$ & $1.5 \pm 0.7$ & $\mathrm{~km} \mathrm{~s}$ \\
\hline & Planet parameters & \\
$M_{\mathrm{P}}$ & $3.22 \pm 0.08$ & $M_{\mathrm{Jup}}$ \\
$R_{\mathrm{P}}$ & $1.02 \pm 0.08$ & $R_{\mathrm{J}}$ \\
$\log g_{\mathrm{P}}$ & $3.89 \pm 0.06$ & $\mathrm{cgs}$ \\
$\rho_{\mathrm{P}}$ & $3.78 \pm 0.06$ & $\mathrm{~g} / \mathrm{cm}^{3}$ \\
\hline & &
\end{tabular}

Fig. 5. Mosaic of the differential light curves obtained during transits of HD 17156b obtained with several telescopes. In each box the horizontal axis is the photometric phase and the vertical axis is the relative flux, along with the best fit model. From left to right column and from top to bottom: $\mathrm{T} 1 \mathrm{a}=$ Almenara, $\mathrm{T} 1 \mathrm{~b}=$ Gasparri and $\mathrm{T} 1 \mathrm{c}=$ Lopresti datasets from the Barbieri et al. (2007). T2a $=\mathrm{OHP}, \mathrm{T} 2 \mathrm{~b}=$ Telast, $\mathrm{T} 2 \mathrm{c}=$ Castellani,$\quad \mathrm{T} 2 \mathrm{~d}=$ Asiago,$\quad \mathrm{T} 2 \mathrm{e}=$ Lopresti,$\quad \mathrm{T} 2 \mathrm{f}=$ Marchini, $\mathrm{T} 2 \mathrm{~g}=$ Nicolini, $\mathrm{T} 2 \mathrm{~h}=$ Papini, $\mathrm{T} 2 \mathrm{i}=$ Vallerani. $\mathrm{T} 2 \mathrm{a}$ to $\mathrm{T} 2 \mathrm{i}$ light-curves were collected on 2007 December 03. T3a $=$ Gary 2007 December 25, $\mathrm{T} 4 \mathrm{a}=$ Gregorio 2008 September 25, T5a = Gary 2008 October 2008.

with other published RV values: F07 (2 datasets: Keck+Subaru), Narita et al. (2008), Cochran et al. (2008) (2 datasets: HET + HJST), we have derived a new spectroscopic orbital solution for HD 17156. A full Keplerian orbit of five parameters: the radial velocity semi-amplitude $K_{\mathrm{RV}}$, the time of periastron passage $\mathrm{T}_{P}$, the orbital period $P$, the orbital eccentricity $e$, and the argument of periastron $\omega$ was adjusted to the data. Second, we carried out a fit to the light-curve that we obtained and also to the Barbieri et al. (2007) datasets using the $e$, and the $\omega$ obtained from the orbital solution. In this scheme, the adjustable parameters are the ratio of the radii $k=R_{\mathrm{p}} / R_{\mathrm{S}}$, their relative sum $\left(R_{\mathrm{s}}+R_{\mathrm{p}}\right) / a$, the orbital inclination $i$, the midtransit time $T_{\mathrm{c}}$, and the orbital period $P$. The values derived from the light-curve analysis were then used to determine, through the analysis of the RossiterMcLaughlin effect, new values of $v \sin i_{\mathrm{S}}$ and of the angle $\beta$ between the equatorial plane of the star and the orbital plane of the planet.

The modeling of the transit lightcurve and RossiterMcLaughlin effect was carried out using the analytical formulae

\subsection{Orbital radial velocity analysis}

In order to derive the stellar spectroscopic orbit using the combined set of radial velocities mentioned above we used only the OOT measurements in all datasets. Observations on the night of the transit are valuable for this because of the steep RV slope (about $23 \mathrm{~m} / \mathrm{s} / \mathrm{h}$ ). We use a downhill simplex algorithm to perform the RV fit to the six datasets, including the zero point shifts between the datasets as free parameters. A stellar jitter of $3 \mathrm{~m} / \mathrm{s}$ was added in quadrature to the observational errors F07.

The best-fit solution has a value of reduced $\chi^{2}=1.13$, and the results are in close agreement with the discovery paper F07 and its subsequent analysis (Irwin et al. 2008). Uncertainties in the best fit parameters were obtained exploring the $\chi^{2}$ grid with adequate resolution. The orbital solution and relative parameter uncertainties are presented in Table 4. In Fig. 7 we show the phased radial velocity curve with the best-fit model. Using the value of primary mass provided in Sect. 3.3 and its uncertainty, the resulting minimum mass for the planet is $m \sin i=3.21 \pm$ $0.08 M_{\mathrm{J}}$, and the semi-major axis is $a=0.1614 \pm 0.0010 \mathrm{AU}$.

\subsection{Photometric analysis}

We used the description of Giménez (2006a) to analyze the lightcurves obtained in Sect. 4.1. In our model we allowed the ratio of the radii, the phase of first contact, the time of transit center and the orbital inclination to vary. We fixed the limb darkening coefficients to the values corresponding to a star with similar temperature and metallicity to HD 17156 from the Claret (2000) tables. For the $R$ band the adopted limb darkening coefficients 


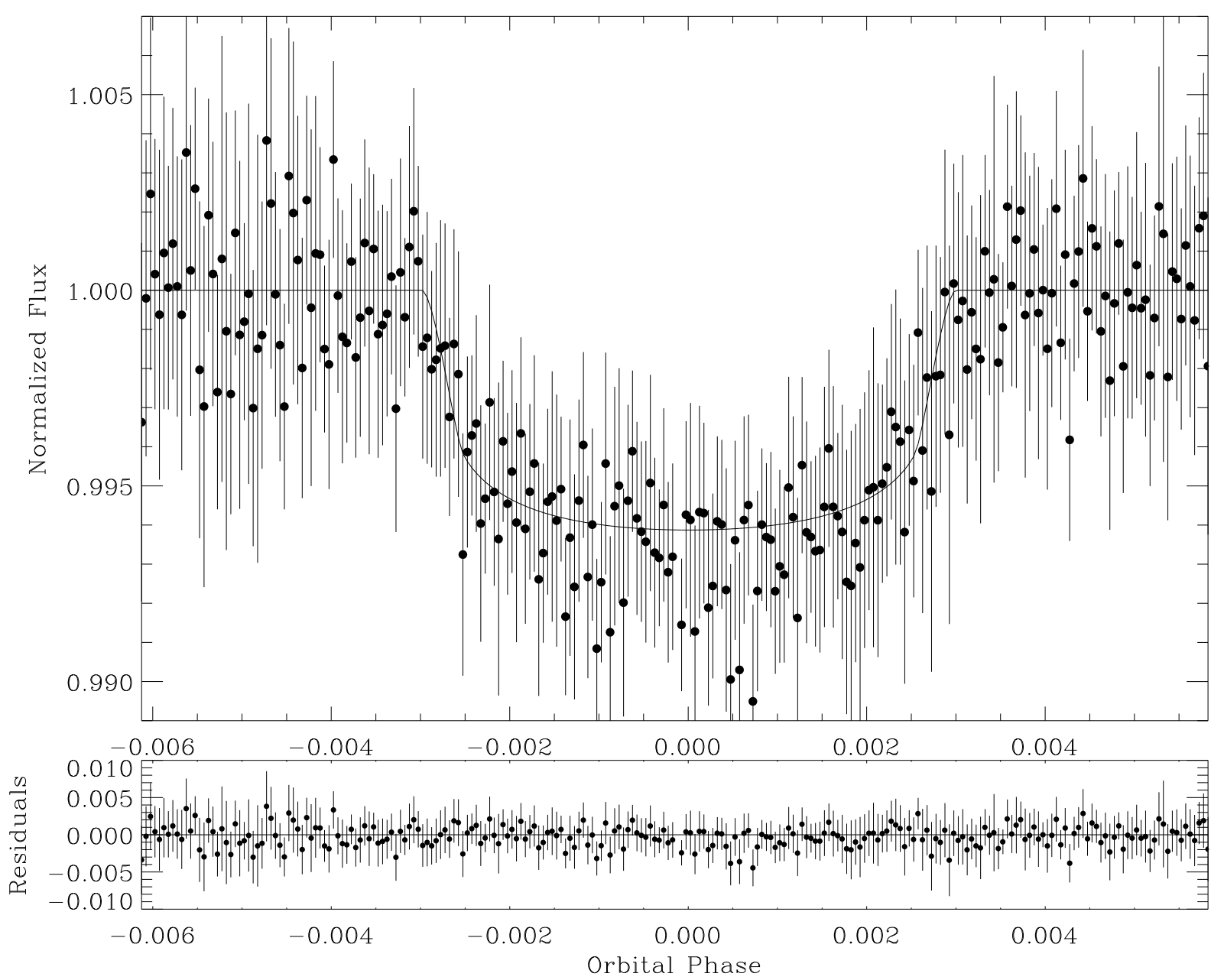

Fig. 6. Combined light curve of all photometric data folded with the orbital period, along with the best fitting model. The time interval between each point is $90 \mathrm{~s}$.

were: $u_{+}=u_{a}+u_{b}=0.6323$ and $u_{-}=u_{a}-u_{b}=-0.0655$. The eccentricity and the longitude of periastron were held fixed to the best fit values obtained from the RV analysis (Sect. 5.1). Errors were estimated using the bootstrap scheme described in Alonso et al. (2008)

The results of the analysis of the 15 datasets are collected in Table 4. Using Kepler's third law we obtain for the stellar and planetary radii $R_{\mathrm{S}}=1.44 \pm 0.08 R_{\odot}$ and $R_{\mathrm{P}}=1.02 \pm 0.08 R_{\mathrm{J}}$.

The histogram of the residuals of the light curves (Fig. 8) has a Gaussian shape with a standard deviation of 0.0062 .

The results are consistent with previous determinations (Barbieri et al. 2007; Irwin et al. 2008; Narita et al. 2008; Gillon et al. 2008); nevertheless the values of inclination and stellar radius show larger deviations than values presented by other authors.

In their analysis Irwin et al. (2008) and Narita et al. (2008) fixed the stellar radius to the value proposed by F07, while Gillon et al. (2008) directly obtained the radius from their analysis. The origin of the discrepancy of the stellar radius might lie in the different values for the inclination, because its value controls the transit duration and the relative sum of the radii. For confirmation we repeated the fit with only the published lightcurve of Gillon et al. (2008) and keeping the limb-darkening coefficients for the $B$ band fixed to $u_{+}=u_{a}+u_{b}=0.7989$ and $u_{-}=u_{a}-u_{b}=0.3019$. The results are the following: $k=0.0729 \pm 0.0031, \theta_{1}=-0.00316 \pm 0.00023, i=87.9 \pm 0.1$, $T_{\mathrm{c}}=2454438.48372 \pm 0.00053 \mathrm{BJD}, R_{\mathrm{S}}=1.44 \pm 0.07 R_{\odot}$ and
$R_{\mathrm{P}}=1.02 \pm 0.07 R_{\mathrm{J}}$. These results are very close to the results of our previous fit.

We note that a transit model that does not take into account the non-zero eccentricity might lead to erroneous results in the orbital inclination and thus also the stellar radius (see, for instance, Sect. 3.2 in Alonso et al. 2008).

\subsection{Rossiter-McLaughlin effect analysis}

The analysis of the TNG RV data obtained during the transit was performed using the formalism developed by Giménez (2006b). We allowed $v \sin i_{\mathrm{S}}$ and $\beta$ to vary and we fixed the values of $K$, $P, e, \omega, k, i, T_{\mathrm{c}}, \theta_{1}$ to the best values obtained from RV and photometry analysis and reported in Table 5. In this analysis we fixed the limb darkening coefficients to the values corresponding to a star with similar temperature and metallicity to HD 17156 from the Claret (2000) tables. The adopted limb darkening coefficients were: $u_{+}=u_{a}+u_{b}=0.7638$ and $u_{-}=u_{a}-u_{b}=0.01828$.

Figure 9 presents the best fitted model to the data.

The best fitted values to the RV orbital residuals of SARG are $v \sin i_{\mathrm{S}}=1.5 \pm 0.7 \mathrm{~km} \mathrm{~s}^{-1}$ and $\beta=4.8^{\circ} \pm 5.3^{\circ}$. The value of $v \sin i_{\mathrm{S}}$ agrees with the values determined by F07 and by our analysis of the stellar spectra. $\beta$ is consistent with zero, indicating that the eclicptic plane of the planet is closely aligned with the equatorial plane of the star. This value of $\beta$ does not confirm the claim of Narita et al. (2008) for a large misalignment in this 

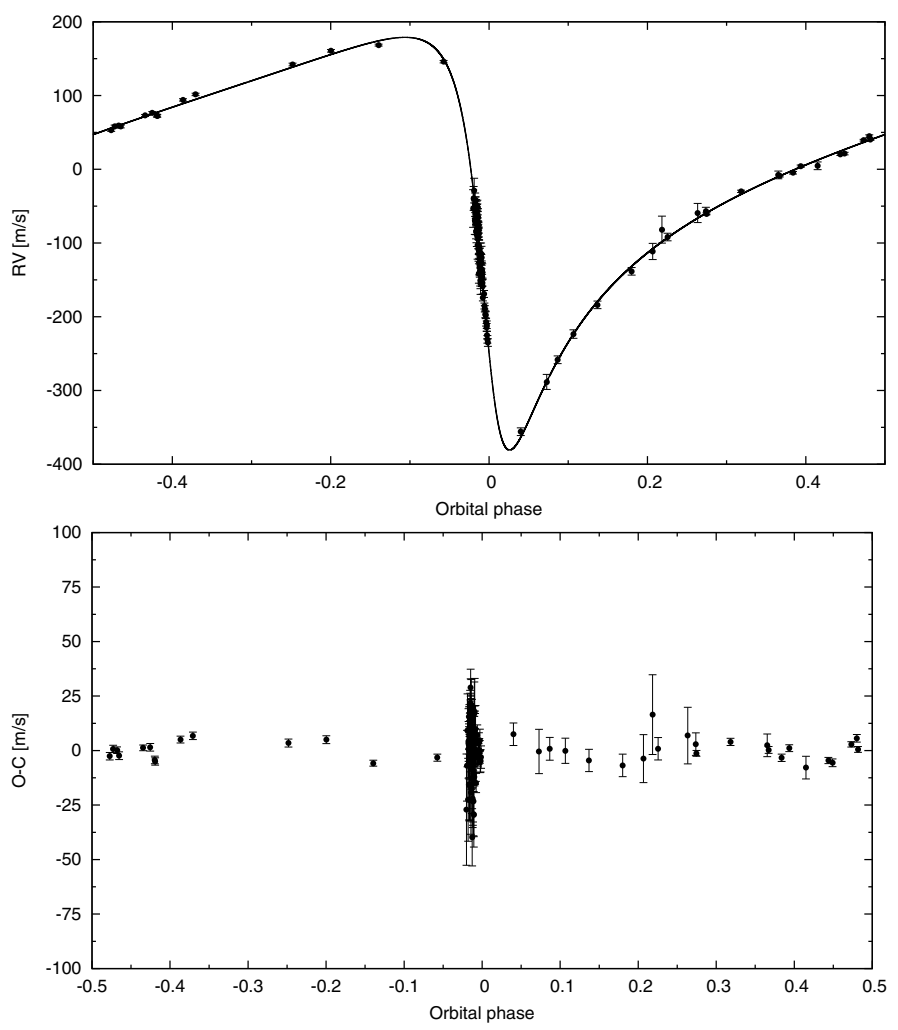

Fig. 7. Upper panel: radial velocities of HD 17156 phased to the best-fit orbital solution. Bottom panel: residuals from the orbital solution.

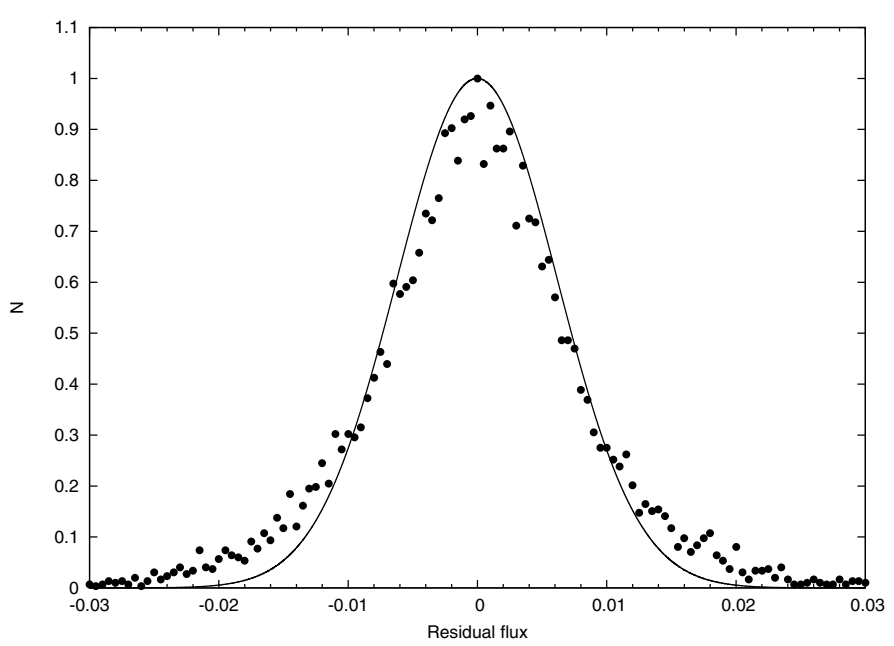

Fig. 8. Histogram of the lightcurve residuals. Overplotted is the best Gaussian fit.

system, but rather agrees with the relative alignment obtained by Cochran et al. (2008). Moreover, the two groups find very different values for $v \sin i_{\mathrm{S}}$. To study the nature of this discrepancy we repeated the fit on their datasets independently, and the results of the fits are summarized in Table 5. The results obtained using the HET dataset, in spite of their good precision, do not provide strong constraints due to their partial coverage of the transit and the fact that the zero point in the OOT data cannot be estimated correctly. Instead, the fit to the HJST data are in excellent agreement with the determination obtained with SARG. Finally, the Narita et al. dataset provides a $v \sin i_{\mathrm{S}}$ in good agreement with previous determinations, and a value of $\beta$ that formally points toward the occurrence of some misalignment. These results
Table 5. Results of the Rossiter-McLaughlin modeling of all datasets. (OAO: Narita et al dataset).

\begin{tabular}{ccccc}
\hline \hline Telescope & Date & $\begin{array}{c}v \sin i_{\text {S }} \\
\mathrm{km} \mathrm{s}^{-1}\end{array}$ & $\begin{array}{c}\beta \\
\mathrm{deg}\end{array}$ & $\chi^{2}$ \\
\hline OAO & $12 / 11 / 2007$ & $1.8 \pm 1.5$ & $-22.6 \pm 20.3$ & 0.7 \\
TNG & $3 / 12 / 2007$ & $1.5 \pm 0.7$ & $4.8 \pm 5.3$ & 1.4 \\
HJST & $25 / 12 / 2007$ & $2.2 \pm 1.0$ & $0.8 \pm 6.4$ & 0.9 \\
HET & $25 / 12 / 2007$ & $1.0 \pm 5.0$ & $30.1 \pm 25.6$ & 0.3 \\
\hline TNG+HJST & & $1.6 \pm 1.0$ & $3.9 \pm 5.4$ & 1.2 \\
\hline
\end{tabular}
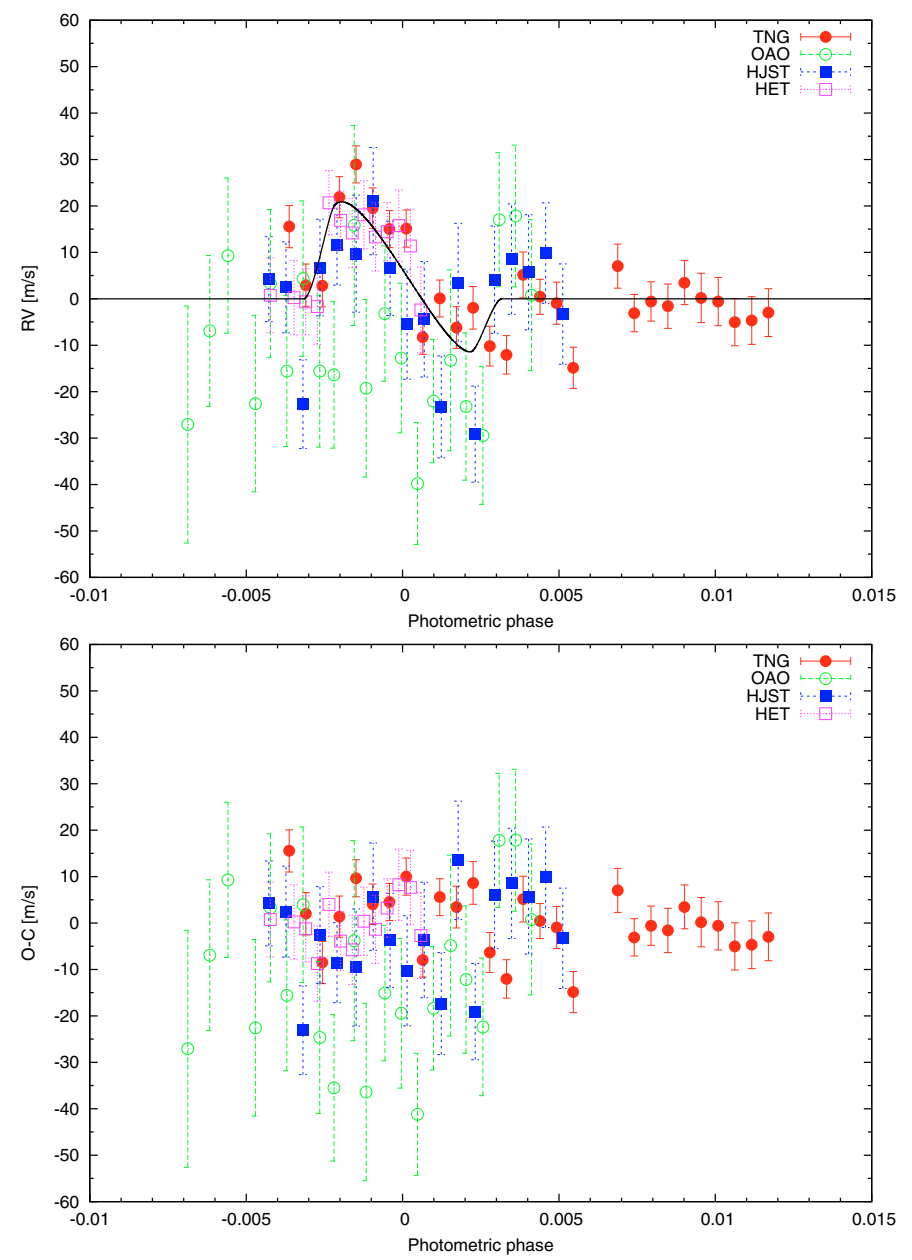

Fig. 9. Upper panel: residuals of radial velocities of HD 17156 phased to the best-fit orbital solution with the best fitted Rossiter-Mclaughlin effect overlayed. Bottom panel: residuals from the Rossiter-Mclaughlin effect.

indicate also that our adopted description is consistent with the one used by Narita et al. however, the intrinsically lower precision of their RV data makes these results not significant.

We also measured line bisectors and bisector velocity span using the technique developed by Martínez Fiorenzano et al. (2005) and looked for changes in the line profiles caused by the planetary transit (see Loeillet et al. 2008). We do not detect significant variations (Fig. 10). This is not unexpected considering the typical signal to noise ratio of our spectra and the low amplitude of the Rossiter signature. 

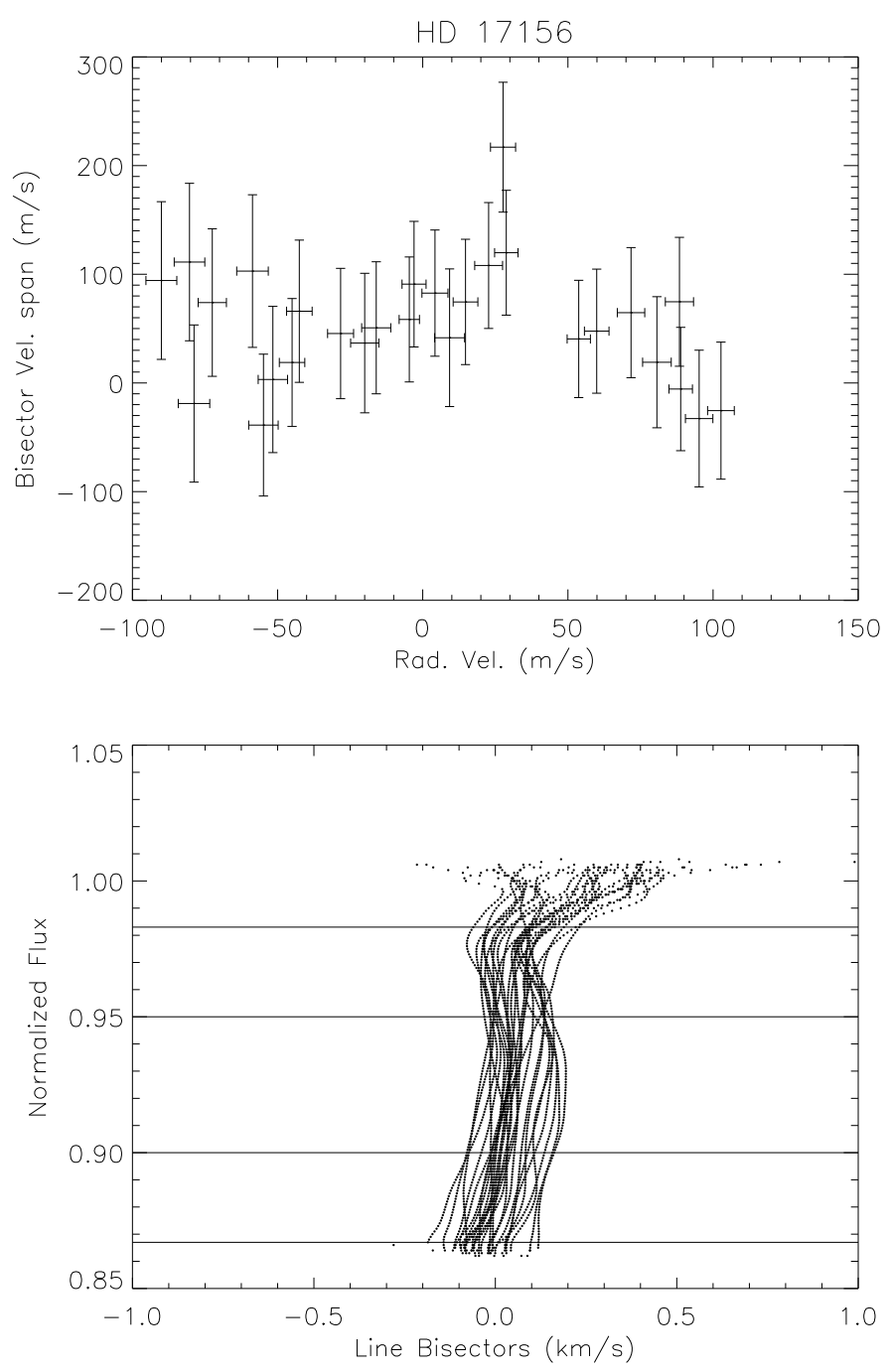

Fig. 10. Bisector velocity span (BVS) from line bisectors of HD 17156 spectra. Upper panel displays BVS vs. RV. Lower panel shows the line bisectors from all spectra, where the horizontal lines enclose the top and bottom zones considered to compute the BVS.

\section{Indications of additional companions}

HD 17156 was observed with the AdOpt module of TNG, the adaptive optics module of TNG (Cecconi et al. 2006). The instrument feeds the $\mathrm{HgCdTe}$ Hawaii $1024 \times 1024$ detector of NICS, the near infrared camera and spectrograph of TNG, providing a field of view of about $44 \times 44$ arcsec, with a pixel scale of $0.0437 \%$ pixel. Plate scale and absolute detector orientation were derived in a companion program of follow-up of binary systems with long term radial velocity trends from the SARG planet search (Desidera et al. 2007).

A series of $15 \mathrm{~s}$ images of HD 17156 were acquired on 3, 18 and 23 October 2007 in the Br $\gamma$ intermediate-band filter. Images were taken moving the target to different positions on the detector, to allow sky subtraction without the need for additional observations, and each night at three different field orientations to make it easier to disentangle true companions from image artifacts. The target itself was used as a reference star for the adaptive optics. Observing conditions were poor on the night of October 3, and rather good on the nights of 18 and 23 October, when we obtained a typical Strehl Ratio of about 0.3 .
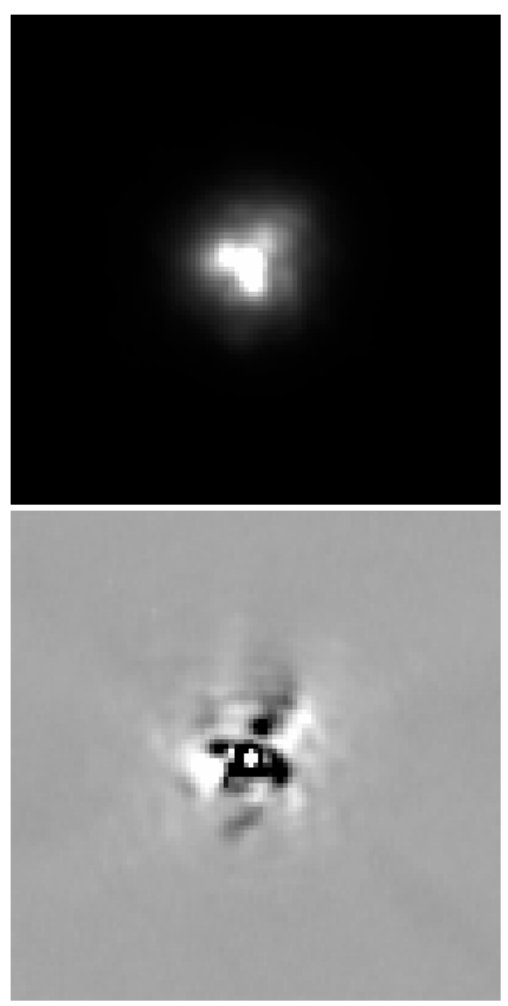

Fig. 11. Images of HD 17156. Upper panel: image of HD 17156 obtained with the AdOpt module of TNG. A similar quasi-static speckle pattern can be seen. Lower panel: difference between two images taken with two different field orientations, which allows us to significantly improve detection limits in the inner regions. The field of view shown is $4.3 \times 4.3^{\prime \prime}$. The images are displayed in linear gray scale.

Data reduction was performed by first correcting for detector cross-talk using dedicated routines ${ }^{5}$ and then performing standard image preprocessing (flat fielding, bad pixel and sky background corrections) in the IRAF environment. Individual images taken at a fixed orientation were shifted and coadded.

The successive analysis was optimized for the detection of companions in different separation ranges. At small separations (from about 0.15 to 2 arcsec) we selected the two best combined images taken at different field orientations on 2007 Oct. 18. They are shown in Fig. 11. These two sets of images are characterized by similar patterns of optical aberrations, and therefore, considering their difference, most of the patterns cancel out in difference images (Fig. 11), improving significantly the detection limits (angular differential imaging, Marois et al. 2006). In the differential image, a true companion is expected to show two peaks, one positive and one negative, at the same projected separation from the central star and position angle displaced by $20^{\circ}$ (the rotation angle between the two sets of images in our case). For detection at separations larger than about 2 arcsec, we summed all the images after an appropriate rotation, obtaining a deep image over a field of about $10 \times 10$ arcsec.

No companion was seen in either the differential image at small separation and or the deep combined image within 10 arcsec. The limit for detection was fixed at peak intensities 5 times higher than the dispersion over annuli at different radial separation. The results both for the differential image and the deep composite image are shown in Fig. 12.

\footnotetext{
5 http://www.tng.iac.es/instruments/nics/files/ crt_nics7.f
} 


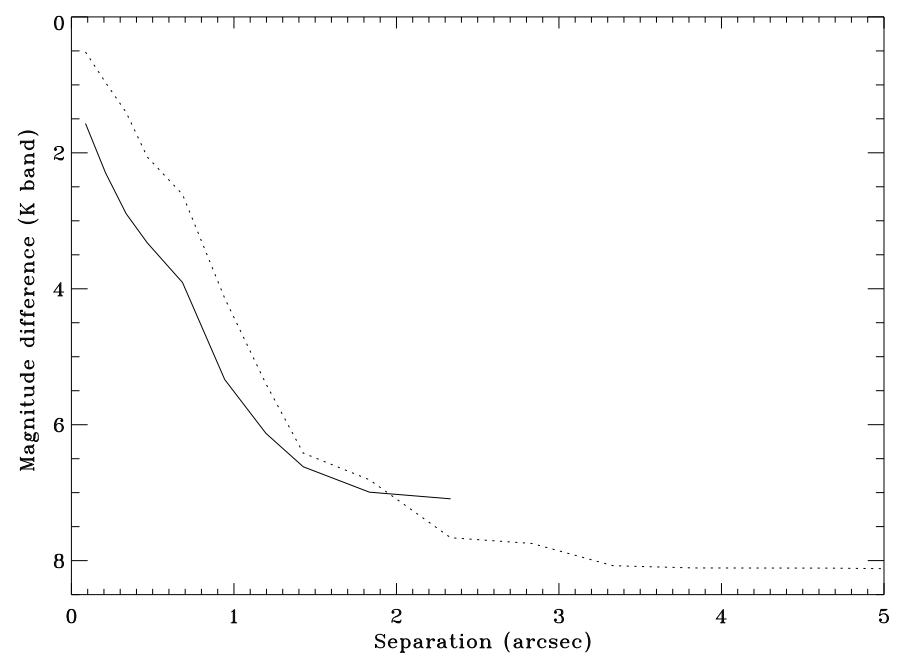

Fig. 12. Detectability limits for companions of difference magnitude around HD 17156 as a function of the projected separation in arcsec. Continuous line: limits on the difference image. Dotted line: limits on the composite deep image.

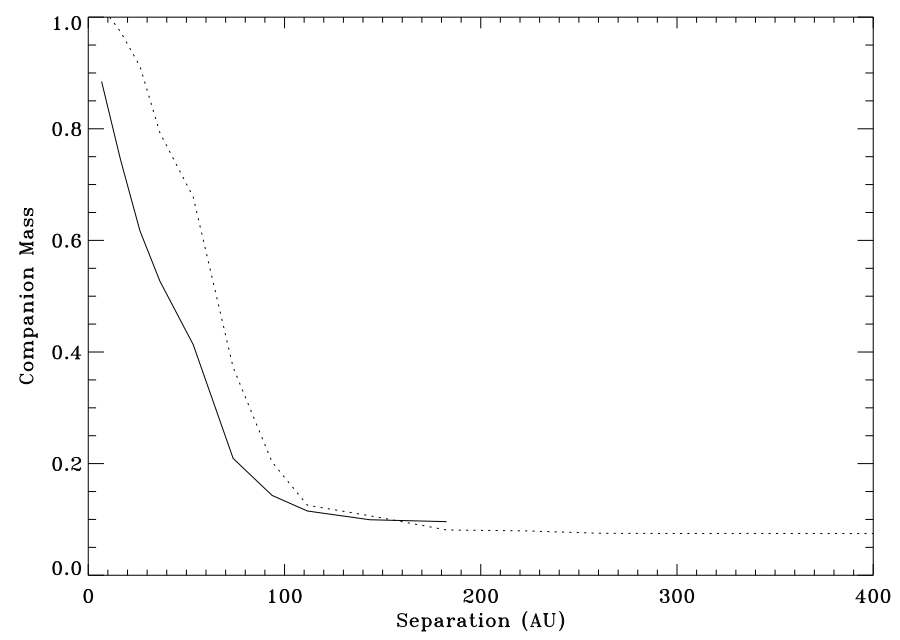

Fig. 13. Detectability limits for stellar companions around HD 17156 as a function of the projected separation in AU. Continuous line: limits on the difference image dotted line: limits on the composite deep image.

The contrast limits derived above were transformed into limits on companion masses using the mass-luminosity relation by Delfosse et al. (2000), and projected separation in arcsec to AU using the Hipparcos distance to the star (Fig. 13). A mainsequence companion can be excluded at a projected separation of between about 150 and $1000 \mathrm{AU}$ (the limit of the image size). At such separations only brown-dwarf or white-dwarf companions are compatible with our detection limits. At smaller separation, detectability worsens quickly, and only stars with mass larger than about $0.4 M_{\odot}$ can be excluded at projected separations closer than $\sim 50 \mathrm{AU}$.

The residuals from the radial velocity orbital solution do not suggest the occurrence of long term trends. This places further constraints on the binarity of the target. However, the timespan is rather short, and the continuation of the radial velocity monitoring is mandatory for a more complete view.

The available astrometric data from Hipparcos does not show evidence for stellar companions either (no astrometric acceleration within the timespan of the Hipparcos observations and no significant differences between Hipparcos and long-term proper motion).

\section{Summary and discussion}

In this paper, we studied the characteristics of HD 17156 and its transiting planets. Stellar parameters (mass, radius, metallicity) agree quite well with the previous study by F07.

Our measurement of the stellar radius of HD 17156 obtained through the analysis of the transit light-curve is the same as the one obtained using the Stefan-Boltzmann law or the Kervella calibration (Table 2). Gillon et al. (2008) obtained a radius of $1.63 \pm 0.2 R_{\odot}$, which is only marginally compatible with our estimate of $1.44 \pm 0.08 R_{\odot}$. On the one hand, to explain such a large stellar radius and the observed visual magnitude, it would be necessary to add $\sim 0.3$ mag of interstellar absorption, that at the distance of HD 17156 is not realistic (suggesting a mean extinction of $4 \mathrm{mag} / \mathrm{kpc}$ ) because HD 17156 is located well inside the Local Bubble, where no strong absorption is present, and the maximum expected absorption is a few hundred mag. On the other hand, the comparison of the Gillon et al. (2008) radius estimate with stellar models also does not appear satisfactory: it is not possible to find a model with a radius that agrees with the observed temperature and metallicity of HD 17156. We conclude that the determination of the stellar radius, and by inference planetary radius, of Gillon et al. (2008) is overestimated by $15 \%$.

For a planet of $3 \mathrm{M}_{\mathrm{J}}$ and an age of $\sim 2 \mathrm{Gyr}$, theoretical models of planet evolution (Baraffe et al. 2008) predict a radius between 0.9 and $1.1 R_{\mathrm{J}}$ as a function of the chemical composition of the planet. Our determination of the radius of HD $17156 \mathrm{~b}$ is $R_{\mathrm{p}}=$ $1.02 \pm 0.08 R_{\mathrm{J}}$. This is in excellent agreement with theoretical expectations. Thus, the strong tidal heating effects on the planet do not appear to contribute to significantly inflate its radius.

Fortney et al. (2007) suggested that HD 17156b, due to its large orbital eccentricity, can change its spectral type during the orbit from a warm $\mathrm{pL}$ type at apoastron to a hotter $\mathrm{pM}$ type at periastron. Their models suggest that for $\mathrm{pM}$ class planets the observed radius int the $R$ band could be 5 larger than the radius measured in the $B$ band (due to the increased opacity of TiO and VO in the $R$ band). Comparing our radius measurements in the $R$ band and the measurements obtained in the $B$ band by Gillon et al. (2008), we find identical results. This result is however not significant because of the large error involved in radius measurement. In order to obtain a significant difference of the radius in $B$ and $R$ band each measurement should be more accurate than $0.03 R_{\mathrm{J}}$.

Our RV monitoring of HD 17156 during the 2007 December 3 transit does not confirm the misalignment between the stellar spin and planet orbit axes claimed by Narita et al. (2008), but it agrees instead with the opposite finding by Cochran et al. (2008). We think our results are more robust because the other most accurate dataset (HET in Cochran et al. 2008) does not cover the full transit, leaving some uncertainties in their Rossiter modeling. We then conclude that the projection on the sky of the stellar spin and planet orbit axes are aligned to better than $10 \mathrm{deg}$.

Therefore HD 17156 joins most of the other exoplanet systems with available measurements of the Rossiter-McLaughlin effect in being compatible with coplanarity. The only possible exception is represented by the XO-3 system, for which Hébrard et al. (2008) found indications for a large departure from coplanarity $\left(\sim 70^{\circ}\right)$. However, as acknowledged by the authors, this result should be taken as preliminary, because of the possibility 
of unrecognized systematic errors in observations taken at large airmass and with significant moonlight contamination.

Our result confirms that large deviations from coplanarity between stellar spin and planet orbit axes are at most rather rare. Such rarity had already been established at a high confidence level for the "classical" Hot Jupiters in short-period circular orbits. For massive eccentric planets the situation is less clear: HD 147506b $\left(M=8.6 M_{\mathrm{J}}, P=5.6\right.$ days, $\left.e=0.52\right)$ and HD $17156\left(M=3.2 M_{\mathrm{J}}, P=21\right.$ days, $\left.e=0.67\right)$ have projected inclinations below $10^{\circ}$ while the possible detection of spin-orbit misalignment in the XO-3 system $\left(M=12.5 M_{\mathrm{J}}, P=3.2\right.$ days, $e=0.29$ ) still awaits confirmation, as discussed above.

The results of the spin-orbit alignment measurements for the HD 17156 system can be compared with the prediction of the planet scattering models. A range of alignments can be the outcome of planet-planet scattering (Marzari \& Weidenschilling 2002). Therefore, our indication of coplanarity does not exclude planet-planet scattering in the HD 17156 system. A larger number of transiting planets with significant eccentricities have to be discovered and characterized to allow more conclusive inferences.

We also searched for stellar companions using adaptive optics, to test the hypothesis of the Kozai mechanism to explain the large eccentricity of HD $17156 \mathrm{~b}$. We did not detect companions within $1000 \mathrm{AU}$, and our detection limits allowed us to exclude main sequence companions with projected separations from about 150 to $1000 \mathrm{AU}$. This result makes unlikely the occurrence of a companion inducing Kozai eccentricity oscillations on the planet, but this possibility cannot yet be completely ruled out (companions at small projected separation and faint white dwarfs and brown dwarf companions are still possible). Continuation of radial velocity and photometric monitoring will allow a more complete analysis of the possible existence of additional companions at small separations.

Acknowledgements. This work was partially funded by PRIN 2006 "From disk to planetary systems: understanding the origin and demographics of solar and extrasolar planetary systems" by INAF. We thank the TNG director for time allocation in Director Discretionary Time. We thank the referre Norio Narita for his useful comments and suggestions.

\section{References}

Allen, C., \& Santillan, A. 1991, Rev. Mex. Astron. Astrofis., 22, 255

Alonso, R., Barbieri, M., Rabus, M., et al. 2008, A\&A, 487, L5

Baraffe, I., Chabrier, G., \& Barman, T. 2008, A\&A, 482, 315

Barbieri, M., Alonso, R., Laughlin, G., et al. 2007, A\&A, 476, L13

Carpenter, J. M. 2001, AJ, 121, 2851

Charbonneau, D., Brown, T. M., Noyes, R. W., \& Gilliland, R. L. 2002, ApJ, 568,377

Charbonneau, D., Brown, T. M., Burrows, A., \& Laughlin, G. 2007, Protostars and Planets V, 701

Cecconi, M., Ghedina, A., Bagnara, P., et al. 2006, Proc. SPIE, 6272, 77

Claret, A. 2000, A\&A, 363, 1081

Cochran, W. D., Redfield, S., Endl, M., \& Cochran, A. L. 2008, ApJ, 683, L59

da Silva, L., Girardi, L., Pasquini, L., et al. 2006, A\&A, 458, 609

Dehnen, W., \& Binney, J. J. 1998, MNRAS, 298, 387

Delfosse, X., Forveille, T., Ségransan, D., et al. 2000, A\&A, 364, 217
Desidera, S., Gratton, R., Endl, M., et al. 2007, ArXiv e-prints, 705, [arXiv: 0705.3141]

Desidera, S., \& Barbieri, M. 2007, A\&A, 462, 345

Endl, M., Kürster, M., \& Els, S. 2000, A\&A, 362, 585

Everhart, E. 1985, Dynamics of Comets: Their Origin and Evolution, Proc. IAU Colloq., 83, ed. A. Carusi, \& G. B. Valsecchi (Dordrecht: Reidel), Astrophys. Space Sci. Lib., 115, 185

Fischer, D. A., Vogt, S. S., Marcy, G. W., et al. 2007, ApJ, 669, 1336

Fortney, J. J., Marley, M. S., \& Barnes, J. W. 2007, ApJ, 659, 1661

Gillon, M., Triaud, A. H. M. J., Mayor, M., et al. 2008, A\&A, 485, 871

Giménez, A. 2006, A\&A, 450, 1231

Giménez, A. 2006, ApJ, 650, 408

Girardi, L., Bressan, A., Bertelli, G., \& Chiosi, C. 2000, A\&AS, 141, 371

Gonzalez, G. 1997, MNRAS, 285, 403

Gonzalez, G. 2008, MNRAS, 386, 928

Gonzalez, G., \& Lambert, D. L. 1996, AJ, 111, 424

Gonzalez, G., \& Vanture, A. D. 1998, A\&A, 339, L29

Gonzalez, G., Laws, C., Tyagi, S., \& Reddy, B. E. 2001, AJ, 121, 432

Gratton, R. G., Bonanno, G., Bruno, P., et al. 2001, Exp. Astron., 12, 107

Hébrard, G., Bouchy, F., Pont, F., et al. 2008, A\&A, 488, 763

Henry, T. J. 2004, Spectroscopically and Spatially Resolving the Components of the Close Binary Stars, 318, 159

Irwin, J., Charbonneau, D., Nutzman, P., et al. 2008, ApJ, 681, 636

Israelian, G., Santos, N. C., Mayor, M., \& Rebolo, R. 2004, A\&A, 414, 601

Johnson, D. R. H., \& Soderblom, D. R. 1987, AJ, 93, 864

Kervella, P., Thévenin, F., Di Folco, E., \& Ségransan, D. 2004, A\&A, 426, 297

Kopal, Z. 1977, Ap\&SS, 50, 225

Kurucz, R. 1993, ATLAS9 Stellar Atmosphere Programs and $2 \mathrm{~km} / \mathrm{s}$ grid. Kurucz CD-ROM No. 13, Cambridge, Mass.: Smithsonian Astrophysical Observatory, 13

Loeillet, B., et al. 2008, A\&A, 481, 529

Mamajek, E. E., \& Hillenbrand, L. A. 2008, ApJ, 687, 1264

Malkov, O. Y. 2003, A\&A, 402, 1055

Malkov, O. Y. 2007, MNRAS, 382, 1073

Marois, C., Lafrenière, D., Doyon, R., Macintosh, B., \& Nadeau, D. 2006, ApJ, 641,556

Martínez Fiorenzano, A. F., Gratton, R. G., Desidera, S., Cosentino, R., \& Endl, M. 2005, A\&A, 442, 775

Marzari, F., \& Weidenschilling, S. J. 2002, Icarus, 156, 570

McLaughlin, D. B. 1924, ApJ, 60, 22

Murray, C. A. 1989, A\&A, 218, 325

Narita, N., Sato, B., Ohshima, O., \& Winn, J. N. 2008, PASJ, 60, L1

Nordström, B., Shporer, A., Bouchy, F., et al. 2004, A\&A, 418, 989

Neuforge-Verheecke, C., \& Magain, P. 1997, A\&A, 328, 261

Nidever, D. L., Marcy, G. W., Butler, R. P., Fischer, D. A., \& Vogt, S. S. 2002 , ApJS, 141, 503

Pasquini, L., Döllinger, M. P., Weiss, A., et al. 2007, A\&A, 473, 979

Ramírez, I., \& Meléndez, J. 2005, ApJ, 626, 446

Reddy, B. E., Lambert, D. L., Laws, C., Gonzalez, G., \& Covey, K. 2002, MNRAS, 335, 1005

Rossiter, R. A. 1924, ApJ, 60, 15

Santos, N. C., Israelian, G., \& Mayor, M. 2004, A\&A, 415, 1153

Santos, N. C., Ecuvillon, A., Israelian, G., et al. 2006, A\&A, 458, 997

Schlesinger, F. 1910, Publications of the Allegheny Observatory of the University of Pittsburgh, 1, 123

Sestito, P., \& Randich, S. 2005, A\&A, 442, 615

Sousa, S. G., Santos, N. C., Israelian, G., Mayor, M., \& Monteiro, M. J. P. F. G. 2007, A\&A, 469, 783

Sozzetti, A., Yong, D., Torres, G., et al. 2004, ApJ, 616, L167

Sozzetti, A., Torres, G., Charbonneau, D., et al. 2007, ApJ, 664, 1190

Sneden, C. A. 1973, Ph.D. Thesis,

Tinetti, G., Vidal-Madjar, A., Liang, M.-C., et al. 2007, Nature, 448, 169

Valenti, J. A., \& Fischer, D. A. 2005, ApJS, 159, 141

Voges, W., Aschenbach, B., Boller, T., et al. 2000, IAU Circ., 7432, 1

van Leeuwen, F. 2007, A\&A, 474, 653

Winn, J. N. 2008, [arXiv: 0807.4929]

Winn, J. N., Holman, M. J., Henry, G. M. et al. 2008, [arXiv: 0810.4725] 